\title{
Experimental Study of Transition Crossing at AGS
}

Jie Wei, BNL, May 4, 1996

\section{Introduction}

II. Results of Experimental Study

* measurement of nonlinear momentumcompaction factor $\alpha_{1}$

* effects of chromatic nonlinearity $* \gamma_{T}$-jump and enhancement of nonlinearity $\left(\alpha_{1}\right)$ $*$ reduction of nonlinearity using sextupoles

III. Comparison with MAD and TIBETAN Simulations

* evaluation of $\alpha_{1}$ and dispersion using MAD * longitudinal simulations using TIBETAN

IV. Conclusions 


\section{Personnel:}

July $27,1994:$

L.A. Ahrens

J.M. Brennan

W.W. Mackay

S. Peggs

T. Satogata

D. Trbojevic

W.K. Van Asselt

A. Warner

J. Wei
Feburary 2, 1995:

L.A. Ahrens

M.M. Blaskiewicz

J.M. Brennan

D-P. Deng

W.K. Van Asselt

J. Wei

Acknowledgements:

E. Gill, M. Harrison, K. Reece, T. Roser,

C. Saltmarsh, M. Syphers, S. Tepikian, ... AGS operation crew 


\section{Introduction}

Transition energy: energy at which particles of different momenta have the same revolution frequency.

(No longitudinal focusing, non-adiabatic synchrotron motion, emittance growth, instabilities, beam loss)

Single-particle effects:

- mismatch in phase switching timing, non-linear bucket

*- chromatic nonlinear effects

Multi-particle effects:

- bunch mismatch due to beam self fields

- combination of self fields and nonlinearity

- microwave instability

Cure:

- avoid transition energy

(un-conventional machine lattice)

* $\gamma_{T}$-jump by pulsing quadrupoles

(distort lattice, enhance $\alpha_{1}$, increase dispersion) 


\section{History:}

- Discovery of the transition energy

N.M. Blackman and E.D. Courant, Rev. Sci. Instr. 20 596 (1949)

- Discussion on chromatic nonlinear effect

K. Jøhnson, Proc. CERN Symp. High-Energy Accel. and Pion Phys. (1956)

- First successful transition crossing on CERN PS and BNL AGS (1960s)

... still, beam loss at $r_{T}$ on AQS.

- Still needs to cross transition in newly designed machines Relativistic Heavy Ion Collider (RHIC)

(superconducting magnets, slow ramping rate, enhanced chromatic effects)

Fermilab Main Injector

- More recent theoretical studies:

K. Takayama, S.Y. Lee, J. Wei, et. al.

- More recent experimental studies:

P. Faugeras, et. al., second order effects in SPS, 1979

J. Wei, M. Brennan, ei. al., experiments done at AGS since 1993 


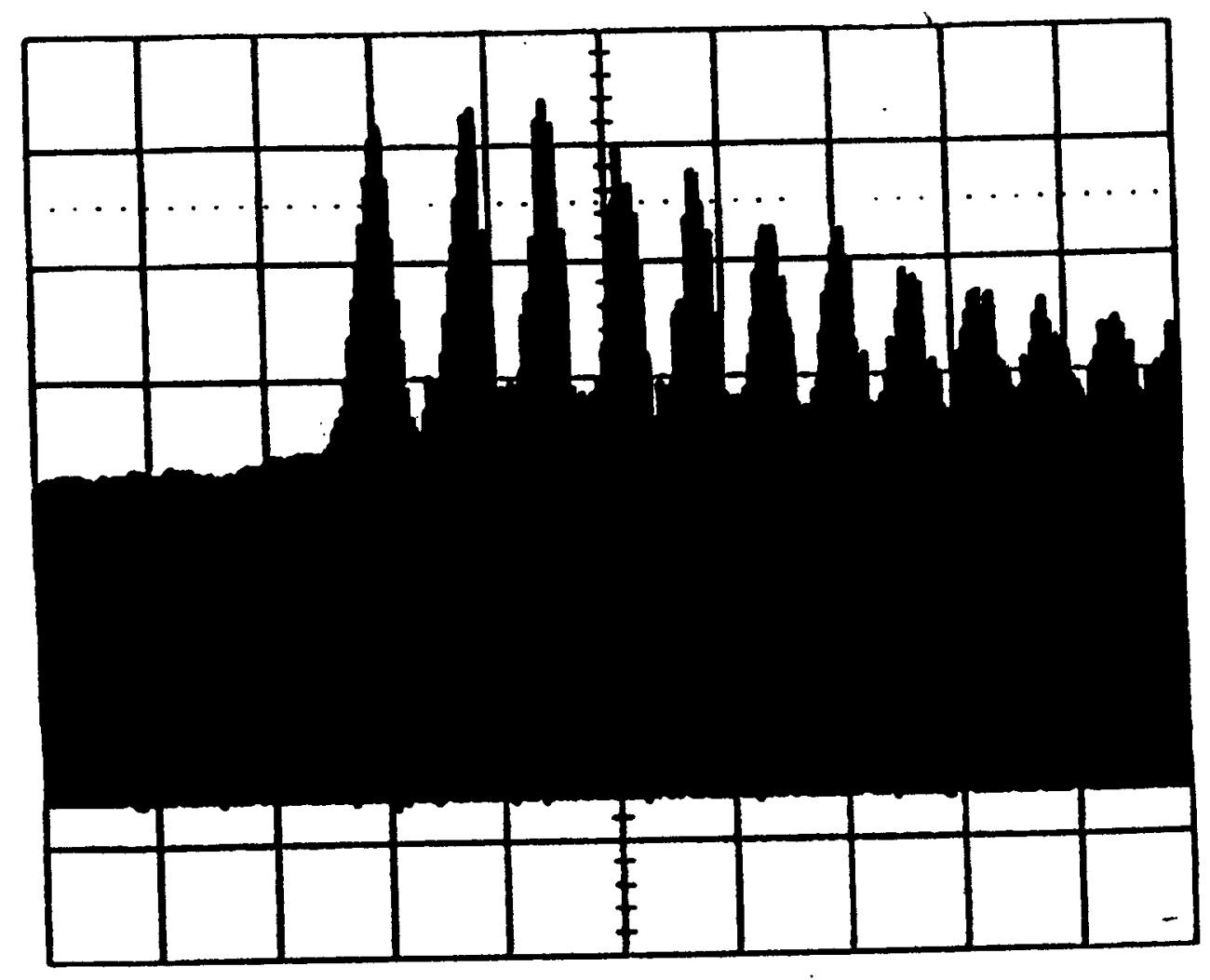

Figure. 1. The envelop of the longitudinal pick-up siganal during transition showing more than $100 \%$ amplitude modulation. The abscissa is time (5 ms per division).

(AGS. Low intensity)

For high intensity proton.

$1.5 T p$ loss at 60Tp intensity 


\section{Results of Experimental Study}

1. Measurement of nonlinear momentum compaction factor $\alpha_{1}$

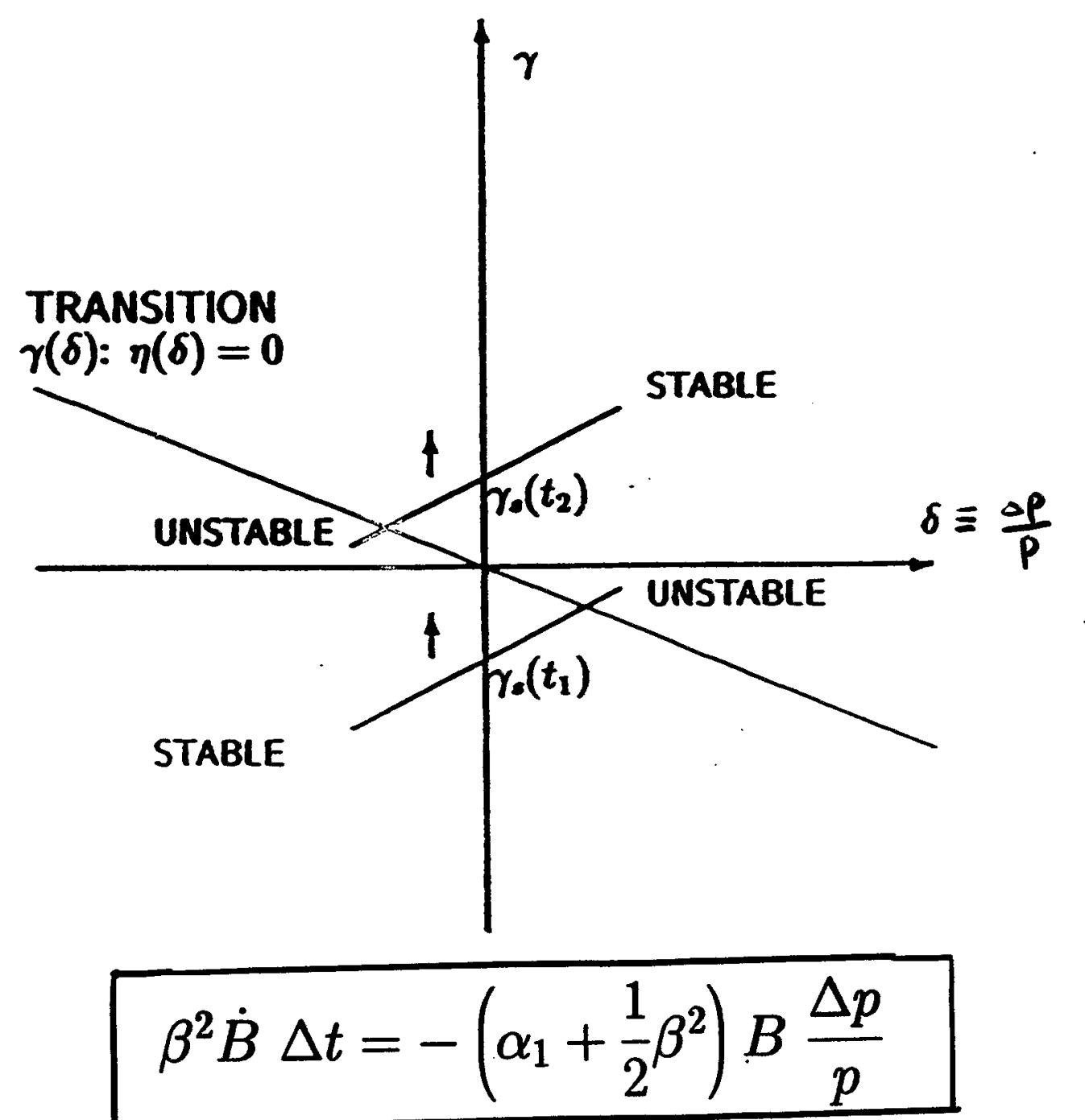

- using "pencil" beam with small $\Delta p / p$;

- vary $\Delta p / p$ by displacing the radial orbit;

- determine transition timing $(\Delta t)$ by measuring the minimum beam loss when varying the time of phase switching.

$$
\frac{\Delta C}{C}=\left[1+\alpha_{1} \delta+\alpha_{2} \delta^{2}+\cdots\right] \cdot \frac{\delta}{\gamma_{T_{0}}^{2}}
$$




$$
\text { 杆 }
$$


Beam loss vs. syn. phase switching time

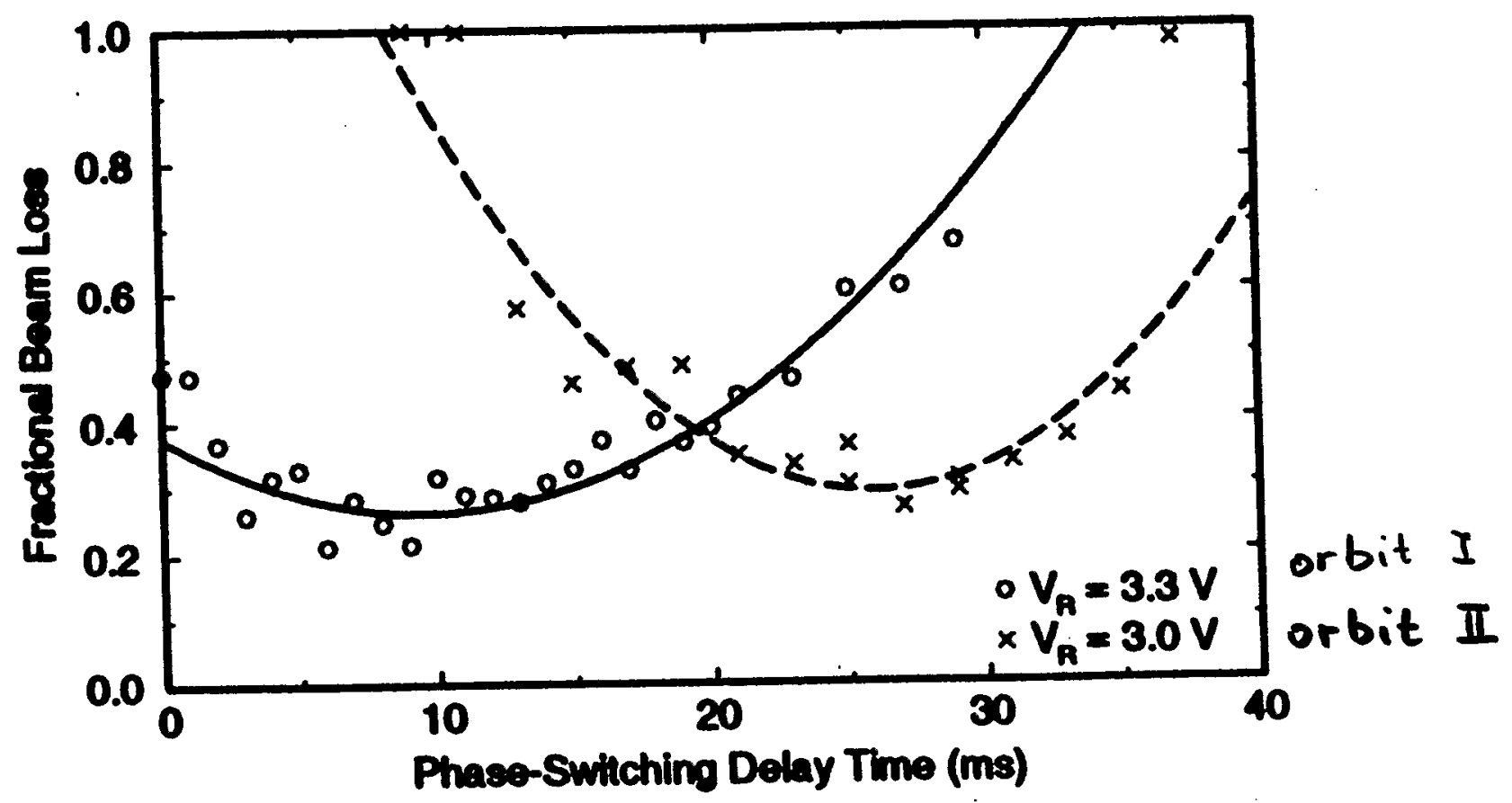

Table 1: Measured AGS $\gamma_{t}, \alpha_{1}$, and momentum aperture at various $\gamma_{t}$-jump quadrupole $\left(I_{Q}\right)$ and sextupole $\left(I_{S}\right)$ settings.

\begin{tabular}{|c|c|c|c|}
\hline$\left(I_{Q}, I_{S}\right)(\mathrm{A})$ & $(0,0)$ & $(1700,0)$ & $(1700,100)$ \\
\hline$\gamma_{t 0}$ & 8.45 & 10.12 & 10.12 \\
$\frac{\alpha_{1}}{\Delta p /\left.p\right|_{\text {ap }}\left(\times 10^{-3}\right)}$ & \pm 7.9 & \pm 4.7 & 16 \\
\hline
\end{tabular}

momentum aperture nominal $Y_{T}$ jump siext. on 
2. Effects of chromatic nonlinearity $\left(\alpha_{1}\right)$
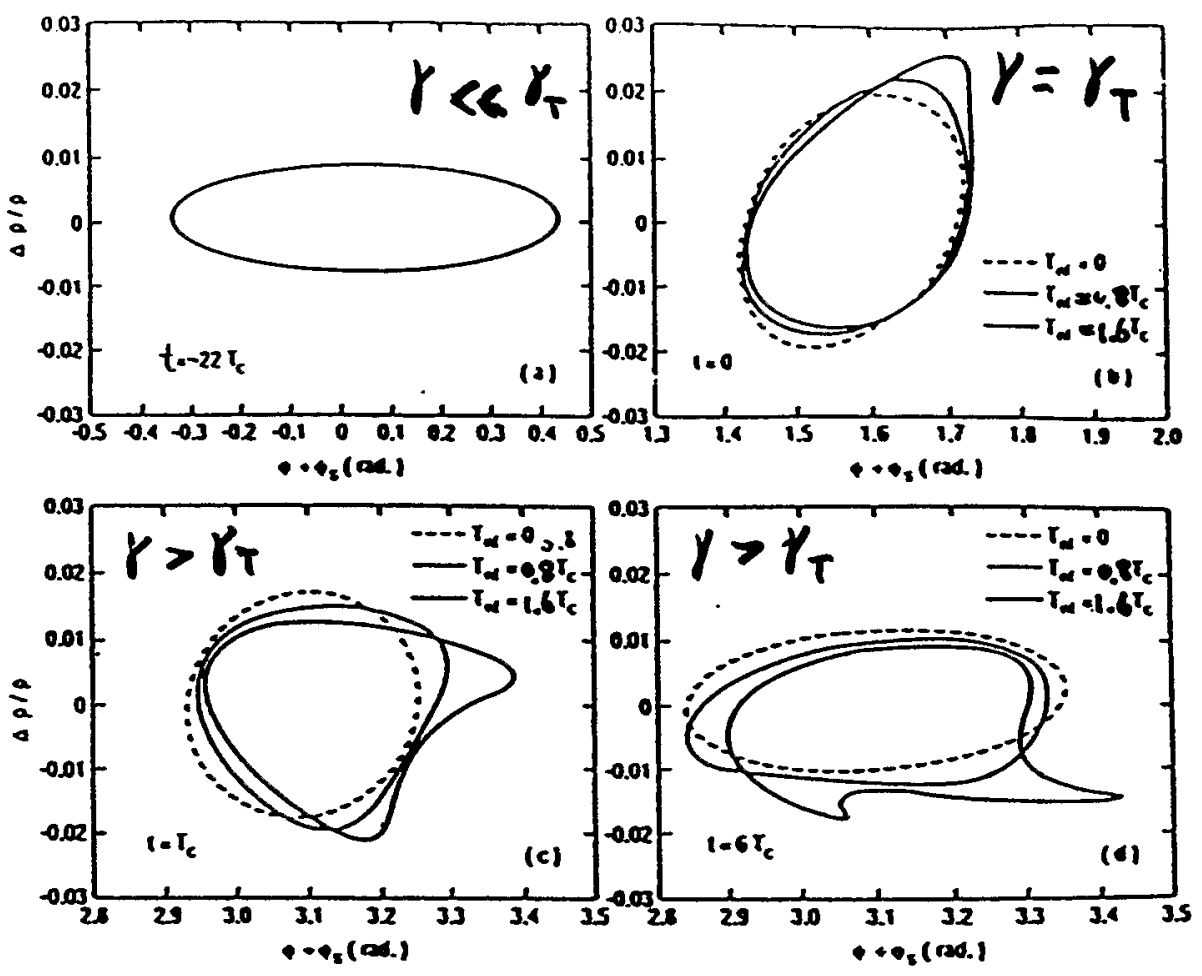

non-adiabatic time:

$$
T_{C}=\left(\frac{\pi E \beta_{s}^{2} \gamma_{T}^{3}}{q e V\left|\cos \phi_{s}\right| \dot{\gamma}_{s} h \omega_{s}^{2}}\right)^{\frac{1}{3}}
$$

nonlinear time:

$$
T_{n l}=\frac{\left|\left(\alpha_{1}+\frac{3}{2} \beta_{s}^{2}\right)\right| \hat{\delta}(0) \gamma_{t 0}}{\dot{\gamma}_{s}}
$$

$$
\frac{\Delta S}{S} \approx\left\{\begin{array}{lr}
0.38 \frac{T_{n l}}{T_{c}}, & \text { for } T_{n l} \ll T_{c} \\
e^{\frac{2{ }^{1 / 2}}{3}\left(\frac{T_{p} l}{T_{c}}\right)^{3 / 2}} & -1, \text { for } T_{n l} \geq T_{c}
\end{array}\right.
$$

(Growth in longitudianl beam emittance) 
* Beam loss vs. crossing rate $\dot{B}$

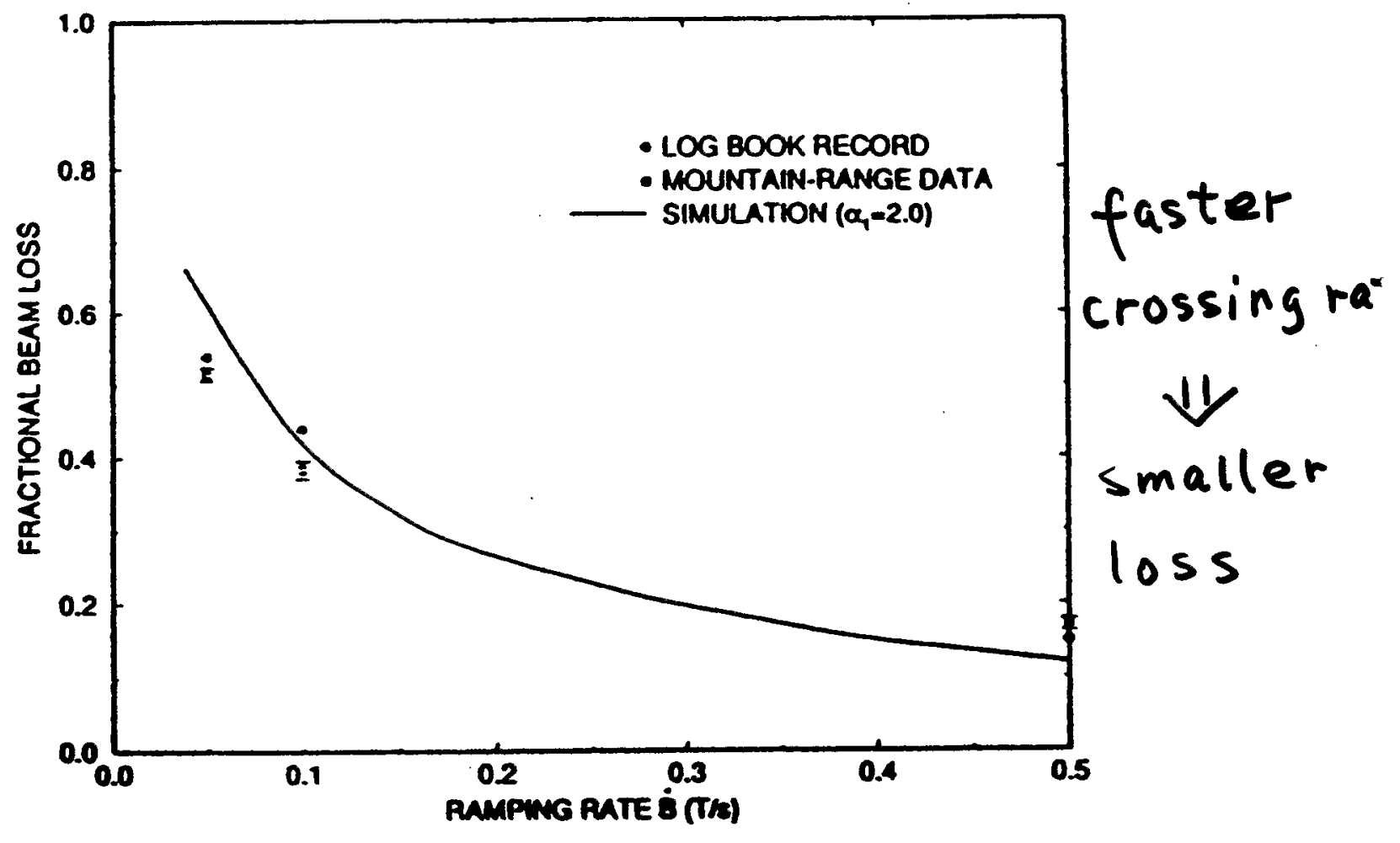

* Beam loss vs. peak rf voltage

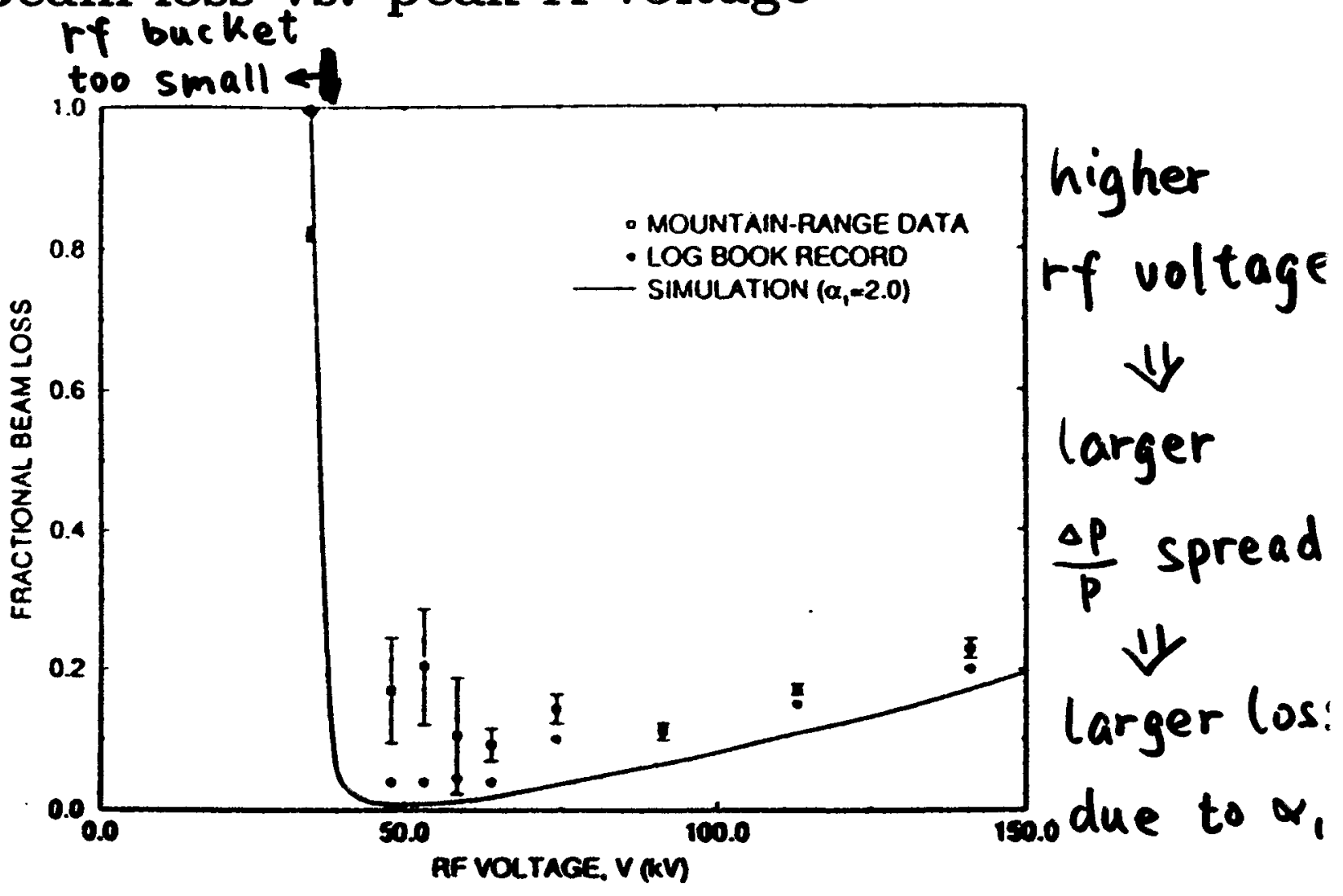


3. $\gamma_{T}$-jump and nonlinearity enhancement

- $\gamma_{T}$-jump improves crossing efficiency by increasing the effective crossing rate

- $\gamma_{T}$-jump usually distorts lattice, enhancing $\alpha_{1}$ and dispersion

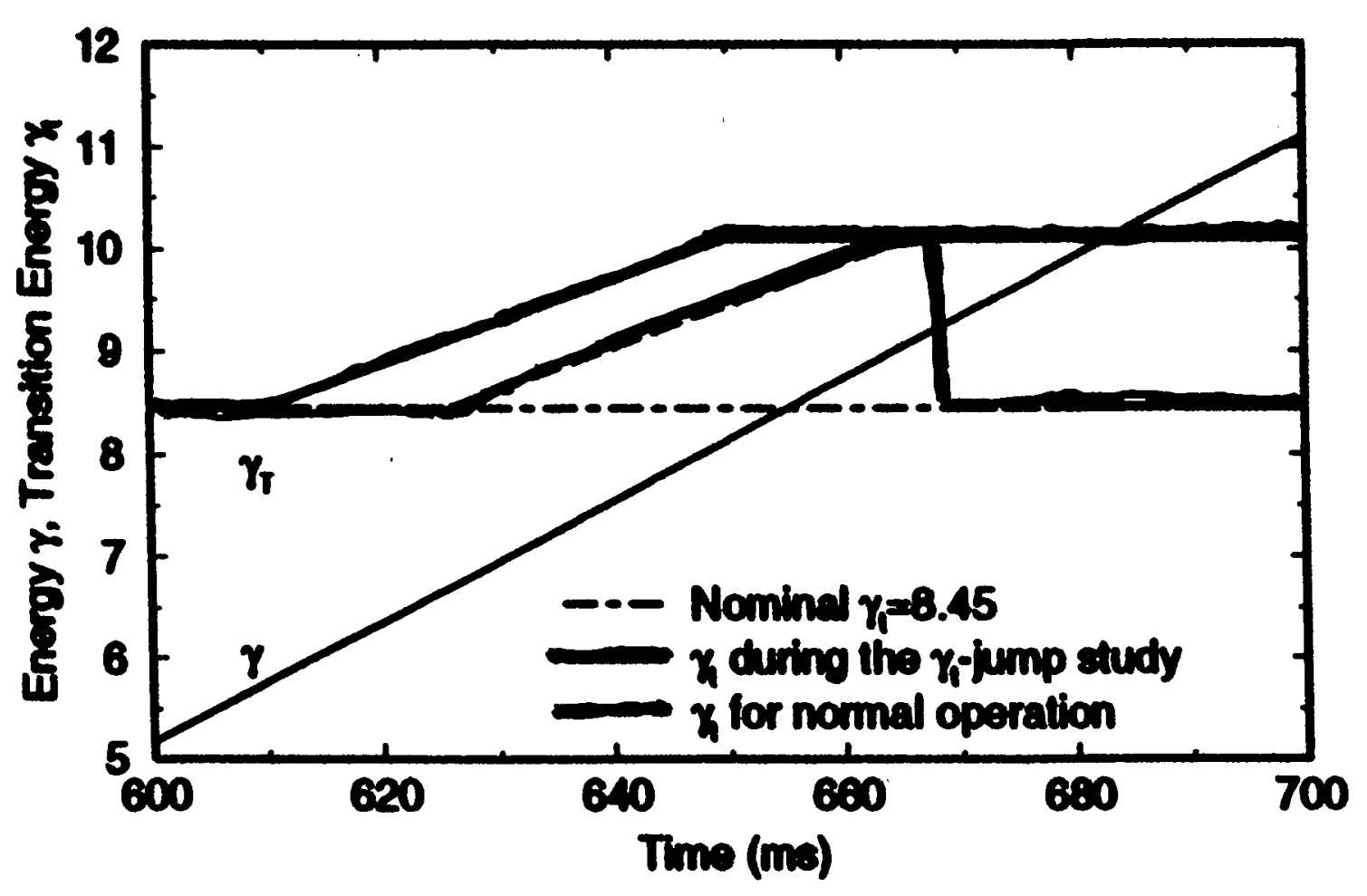


4. reduction of nonlinearity using sextupoles

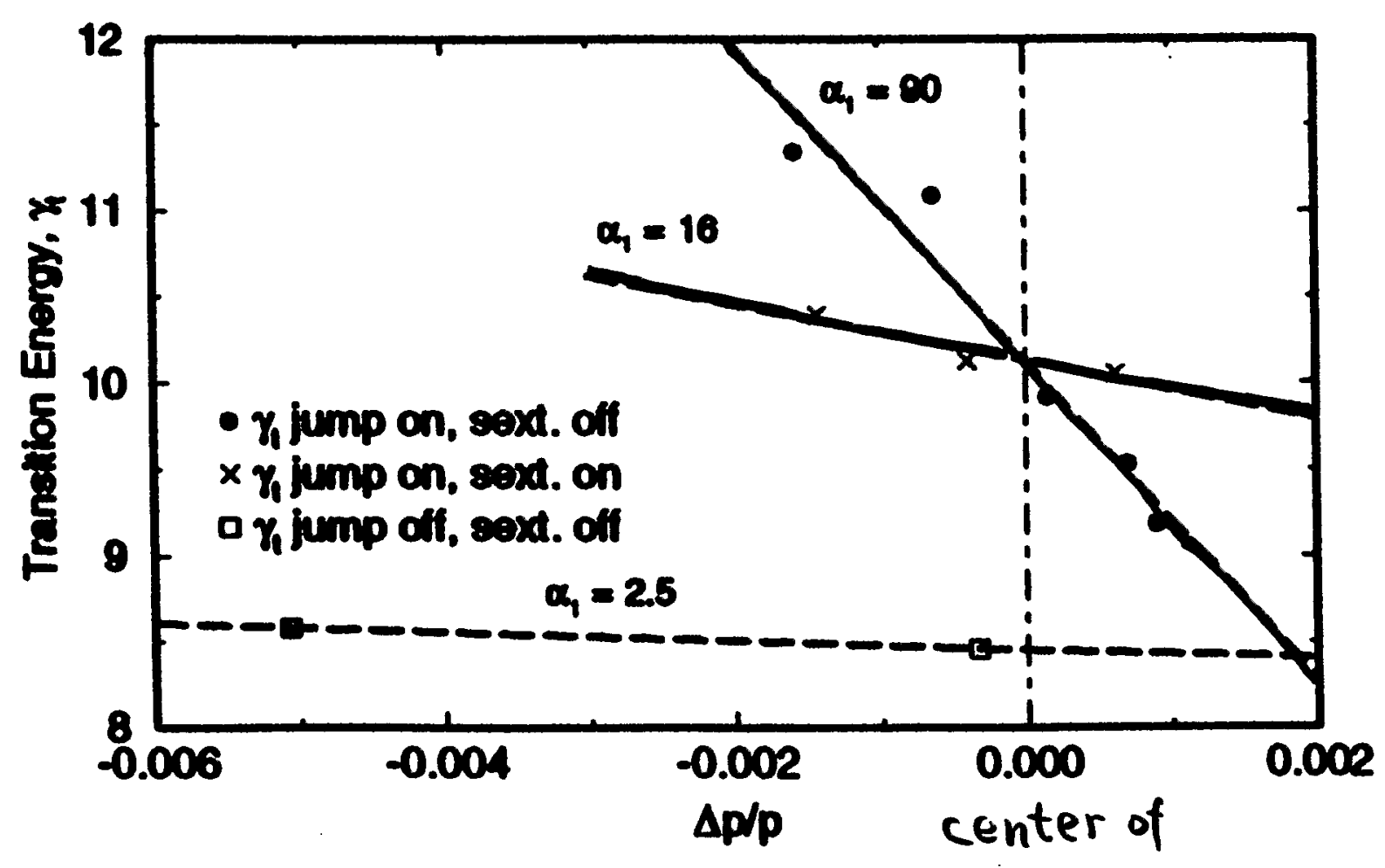

sextupoles

nominal operation: $\alpha_{1}=2.5$.

aperture $\frac{\Delta p}{p}= \pm 0.8 \%$

$\gamma_{T}$ jump lattice: $\quad \alpha_{1}=90$

aperture $\frac{\Delta p}{p}= \pm 0.5 \%$

sextupoles on : $\alpha_{1}=16$

aperture $\frac{\Delta p}{p}= \pm 0.4 \%$ 


\section{Comparison with MAD and TIBETAN Simulations}

1. $\alpha_{1}$ and dispersion evaluation using MAD

Table 2: MAD calculation of AGS $\gamma_{t 0}, \alpha_{1}, \alpha_{2}$ and maximum dispersion $\left.\eta_{x}\right|_{\max }$ at the $\gamma_{t}$-jump quadrupole and sextupole settings corresponding to Table 1.

nominal $\gamma_{T}$-jump sext. on

\begin{tabular}{|c|c|c|c|}
\hline$\left(I_{Q}, I_{S}\right)(\mathrm{A})$ & $(0,0)$ & $(1700,0)$ & $(1700,100)$ \\
\hline$\gamma_{t 0}$ & 8.45 & 10.12 & 10.12 \\
$\alpha_{1}$ & 2.2 & 76 & 19 \\
$\alpha_{2}$ & 8.9 & $-2.7 \times 10^{3}$ & $-1.6 \times 10^{3}$ \\
$\left.\eta_{x}\right|_{\max }(\mathrm{m})$ & 2.2 & 8.6 & 8.6 \\
\hline
\end{tabular}

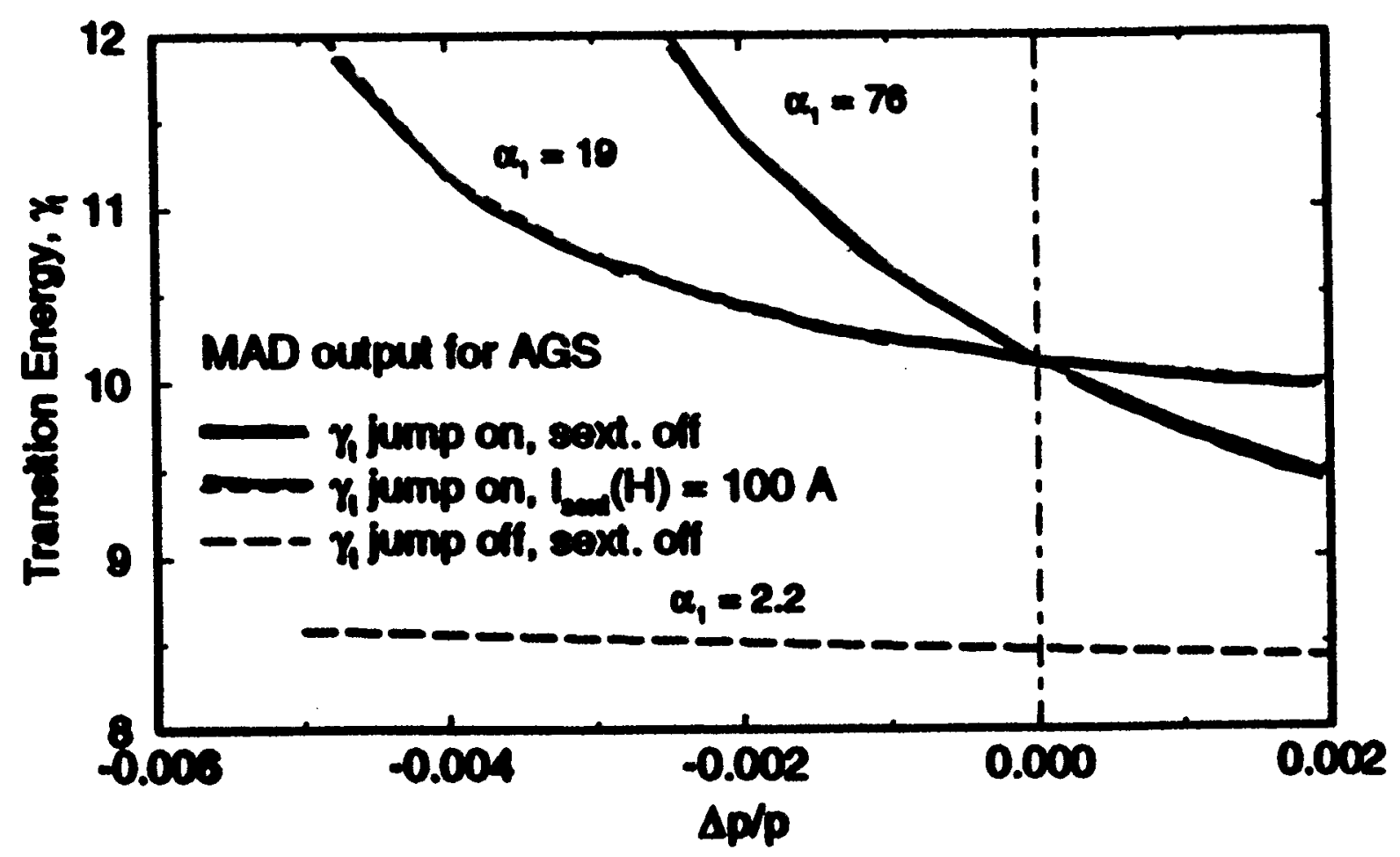


2. Longitudinal simulation using TIBETAN

- Using experimentally extracted $\alpha_{1}$ and machine parameters, simulate transition crossing using TIBETAN under the same experimental condition

- Compare simulated mountain-range plots with experimental digitized beam profile data, using the same post-analysis codes (GT_ANALY)

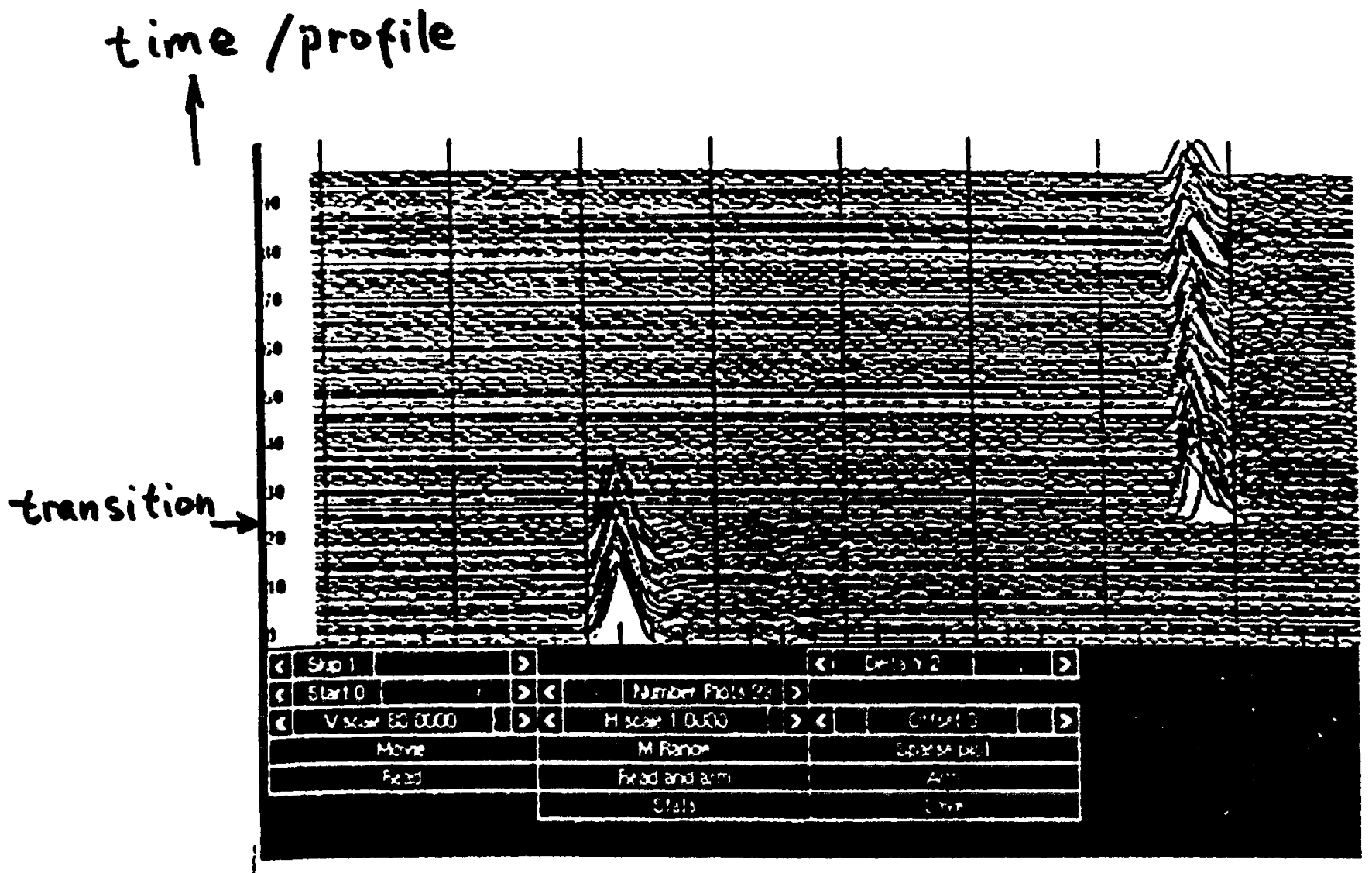

Figure : Typical digitized beam-profile data. 


\section{Conclusions}

- Although $\gamma_{T}$-jump in AGS improves transition crossing efficiency for high intensity beams, it enhances chromatic nonlinear effects $\left(\alpha_{1}\right)$.

- The sextupoles can be excited to greatly reduce $\alpha_{1}$, hence improving longitudinal crossing at transition. However, the current scheme results in large dispersion.

- An optimization in $\gamma_{T}$-jump scheme and sextupole arrangement can greatly improve AGS operation at transition. 


\section{References}

1. K. Jøhnsen, Proc. CERN Symp. High-Energy Accel. and Pion Physics (Geneva, 1956), Vol.1, p.106.

2. K. Takayama, Part. Accel. 14, 201 (1984).

3. W. Hardt, et al., Proc. 7th Int. Conf. on High-Energy Accel., Yereran, 329 (1969).

4. E. Ciapala, et al., IEEE Trans. Nucl. Sci. NS-26, 3571 (1979).

5. P. Faugeras, et al., IEEE Trans. Nucl. Sci. NS-26, 3577 (1979).

6. S.Y. Lee and J. Wei, EPAC Proc. (Rome, 1988), p.764.

7. J. Wei, Ph.D thesis (1990), revised Nov. 1994.

8. J. Wei, Proc. 3rd EPAC, Berlin, 643 (1992).

9. J. Wei, A. Warner, et al., EPAC 1994, London, p.976 (1994).

10. L.C. Teng, FN-207/400 (FNAL, Batavia, 1970).

11. L. Thorndahl, ISR-300/LI/69-38 (CERN, Geneva, 1969).

12. A. Sørenssen, Part. Accel. 6, 141 (1975).

13. L. Ahrens, et al., AD/No.265 (BNL, 1986).

14. S.Y. Lee and K.Y. Ng, Proc. Fermilab III Instab. Workshop, 170 (1990).

15. P. Yamin, et al., Proc. 1987 IEEE Part. Accel. Conf., 87CH23879, p.194.

16. W.K. van Asselt, Proc. 1995 Particle Accel. Conf. (to be published).

17. M. Brennan, Proc. 1995 Particle Accel. Conf. (to be published).

18. H. Grote et al., MAD 8.13, CERN/SL/90-13 (1990).

19. J.P. Shan, et al., Particle Accelerators 451 (1994).

20. J. Woi, J.M. Brennan, et. al., Proc. 1995 Particle Accel. Conf. (to be Dublished). 